\title{
Integrating prior knowledge and locally varying parameters with Moving-GeoStatistics: methodology and application to bathymetric mapping
}

\author{
Cédric MAGNERON \\ ESTIMAGES, 10 Avenue du Québec, 91140 Villebon-sur-Yvette - France \\ +33160924121 \\ cedric.magneron@estimages.com
}

Nicolas JEANNEE

GEOVARIANCES, 49bis Avenue Franklin Roosevelt, BP91, 77212 Avon France

+33160747454

jeannee@geovariances.com

Olivier LE MOINE

IFREMER, , Laboratoire Environnement-Ressource des Pertuis Charentais, Avenue de Mus de Loup, 17390 La Tremblade - France

+33546762621

olemoine@ifremer.fr

Jean-François BOURILLET

IFREMER, Dép. Géosciences Marines, Laboratoire Environnements

Sédimentaires, BP70, 29280 Plouzané - France

+33298224243

Jean.Francois.Bourillet@ifremer.fr

Keywords: Moving-GeoStatistics, variogram-based models optimization, stationarity, local anisotropies, varying scale structures, bathymetry mapping. 


\title{
Table of contents
}

\author{
Abstract \\ Introduction \\ 1. Conventional variogram-based models \\ 1.1. Global approach \\ 1.2. Variogram-based models parameters \\ 1.3. Limits \\ 2. M-GS (Moving-GeoStatistics) models \\ 2.1. Principle \\ 2.2. M-parameters \\ 2.3. Advantages \\ 3. M-GS application to bathymetric mapping \\ 3.1. Context \\ 3.2. Data set description \\ 3.3. Conventional variogram-based mapping \\ 3.4. M-GS mapping \\ Conclusion \\ Acknowledgments \\ References
}

\section{Abstract}

Most geostatistical methods rely on a global variogram model, assuming stationarity for the underlying random function. Applying stationary approaches in the case of large/complex areas, even locally with a moving neighbourhood, can lead to unsuitable estimates. Though preferable to some extent, non stationary approaches hardly handle prior knowledge nor reproduce precisely complex structures, such as local anisotropies, spatially varying small-scale structures or heterogeneity.

The paper aims at presenting an innovative methodology, called M-GS (MovingGeoStatistics), which is fully dedicated to the local optimization of parameters involved in variogram-based models. M-GS considers the structural and computational parameters as a set of dependant parameters to be spatially optimized. The optimization process, which may be guided by objective or subjective criteria, is carried out during a M-structural analysis phase that leads to a set of spatially variable structural and computational parameters. Thus, M-GS ensures a better adequacy between the geostatistical model and the data. 
The methodology is applied for bathymetry mapping. The adequacy of the M-GS methodology is illustrated and compared with classical estimates for the MarenneOléron coast (West of France).

\section{Introduction}

Today, most geostatistical methods rely on a global variogram model. The variogram allows to build effective estimation (kriging) and simulation operators by catching the mean spatial correlation inherent to a data set. These methods commonly assume stationarity for the underlying random function. This assumption is too constraining in numerous applications, as soon as the target area becomes large or involves complex structural patterns. Applying stationary approaches in such cases, even locally with a moving neighbourhood, can lead to unsuitable estimates and non stationary approaches are preferable to some extent, provided that one is ready to accept to loose some control on the underlying structural model. Furthermore, even non stationary algorithms hardly handle prior knowledge nor reproduce precisely complex structures, such as local anisotropies, spatially varying small-scale structures or heterogeneity, etc.

The paper aims at presenting an innovative methodology, called M-GS (MovingGeoStatistics), which is fully dedicated to the local optimization of parameters involved in variogram-based models. M-GS considers the structural and computational parameters as a set of dependant parameters to be spatially optimized. The optimization process, which may be guided by objective or subjective criteria, is carried out during a M-structural analysis phase that leads to a set of spatially variable structural and computational parameters. Thus, M-GS ensures a better adequacy between the geostatistical model and the data.

The methodology is applied for bathymetry mapping. The adequacy of the M-GS methodology in a complex structural environment and for a specific orientedacquisition design is illustrated and compared with classical estimates for the Marenne-Oléron coast (West of France). 


\section{Conventional variogram-based models}

\subsection{Global approach}

The majority of geostatistical models that are daily implemented in the industry are variogram-based models - see (Dubrule 2003) for example. They are used for processing spatially distributed data, especially in natural resources domains such as mines, petroleum and environment. They are mainly devoted to mapping, filtering and uncertainty management applications.

Variogram-based models rely generally on the modelling of a statistical function, the experimental variogram, which depicts the mean spatial correlation between data samples. When data can be considered as the result of a stationary random process, the variogram model is fitted directly to the experimental variogram, which is supposed to be representative of the whole data field or of a wellseparated area of the data field. Based on the variogram model, effective estimation (kriging) and simulation operators are built and applied to the data set.

In the second-order stationary case, the variogram-based approach is rather intuitive as some parameters of the model may be related directly to the observation of the data themselves. Non-stationary models, such as IRF-k models (Matheron 1971, Chilès 1999), are more intricate and lead to less control on the underlying structural model. It justifies that, very often, data are still transformed for working in a stationary framework as in the universal kriging case, despite the observed bias of the residuals variogram (Pardo-Igúzquiza 1998).

\subsection{Variogram-based models parameters}

\subsubsection{Structural parameters}

In the stationary case variogram modelling is driven through a two-steps phase called structural analysis. The first step consists in interpreting the experimental variogram computed from the data. This step is rather likely to involve the user's knowledge about his data set. Based on the first step conclusions, the second step aims at fitting a single or a set of parameterized functions to the experimental 
variogram, thus defining the variogram model. Broadly speaking, structural parameters are the parameters that are related to the variogram model such as range(s), sill(s), anisotropy coefficient(s), etc.

\subsubsection{Computational parameters}

In order to run variogram-based estimation and simulation algorithms, some computational parameters must be tuned. They are mainly tied to the moving neighbourhood used for selecting data points surrounding the target point (the point to be estimated or simulated). In practice, the computational parameters are often utilized for managing processing times, specifically when dealing with large data sets, or for adjusting the neighbourhood according to the samples pattern.

\subsection{Limits}

Variogram-based estimation and simulation results are sensible to structural and computational parameters. Although sensibility may be highly variable depending on some data characteristics, such as sampling density or variable continuity for example, it is rarely null. This point is often unappreciated while running variogram-based models.

More specifically, sensibility to the parameters can be very problematic when facing with complex structural environment or specific acquisition patterns. In such cases global stationary models may not fit to local data characteristics and can lead to unexpected poor results.

\section{M-GS (Moving-GeoStatistics) models}

\subsection{Principle}

M-GS methodology is fully dedicated to the local optimization of parameters involved in variogram-based models. M-GS considers the structural and computational parameters as a set of dependant parameters to be spatially optimized. The optimization process, which may be guided by objective or 
subjective criteria, is carried out during a M-structural analysis phase that leads to a set of spatially variable structural and computational parameters.

\subsection{M-parameters}

M-parameters are locally optimized versions of structural and computational parameters of variogram-based models. They vary spatially over the data field. In the past, non-stationarity has been explored for several parameters, such as anisotropy, especially in the environment domain (see Caetano 2004 for example). When dealing with these models the major challenge is to get stable variations of the parameters and as far as possible to automate the parameters determination process.

Several approaches are possible to compute M-parameters. A simple one merely consists in computing local variogram parameters in adjacent areas of the data field and then to smooth the obtained parameters in order to make them available at every target grid node. More sophisticate algorithms currently under development are based on automatic validation techniques. They simplify the determination of the M-parameters and lead to promising results on various real cases that have been tested.

One example of results obtained with an automatic validation approach is presented in Fig_ch2_ 1, which displays a 2D seismic data set (Fig_ch2_ 1a) and one associated M-parameter map corresponding to the range variations of an isotropic spherical model (Fig_ch2_1b). An interpolation error criterion has been used for determining the optimal parameters. The north-eastern part of the data field appears to be less structured (range smaller) than the rest of the data field. The M-parameters are used to map the seismic data by ordinary kriging (Fig_ch2_1c).

It should be noted that the M-structural analysis process involves some dependency relationship between several parameters. For example, in the secondorder stationary case the size of the moving neigbourhood in one direction is 
related linearly to the range of the largest scale structure in that direction. More complex relationships can be introduced into the optimization process.

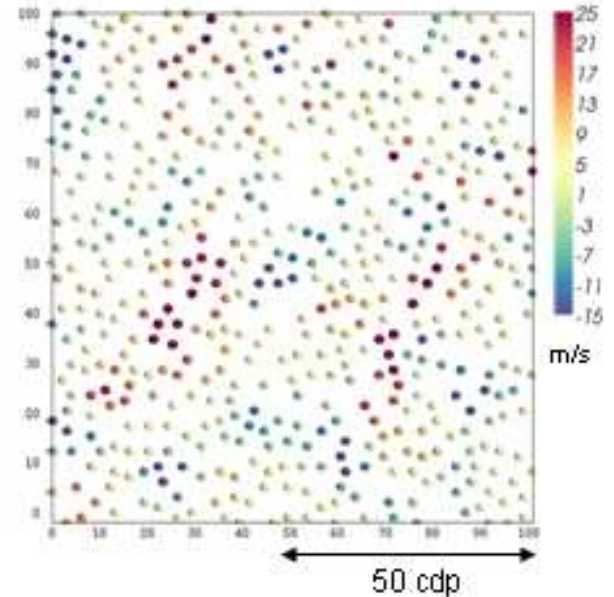

Fig_ch2_1a

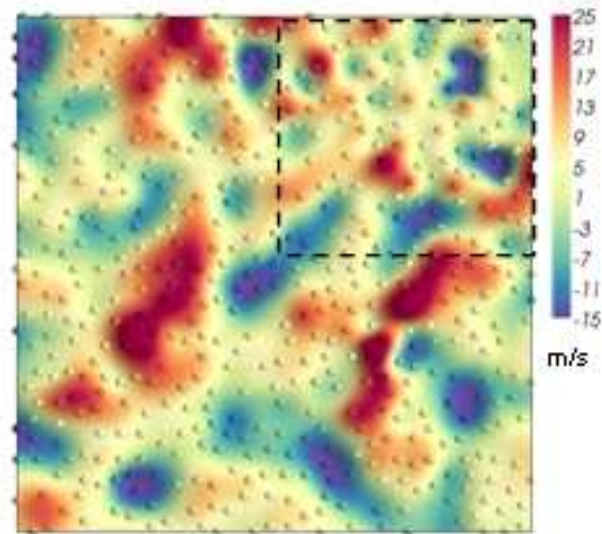

Fig_ch2_1c

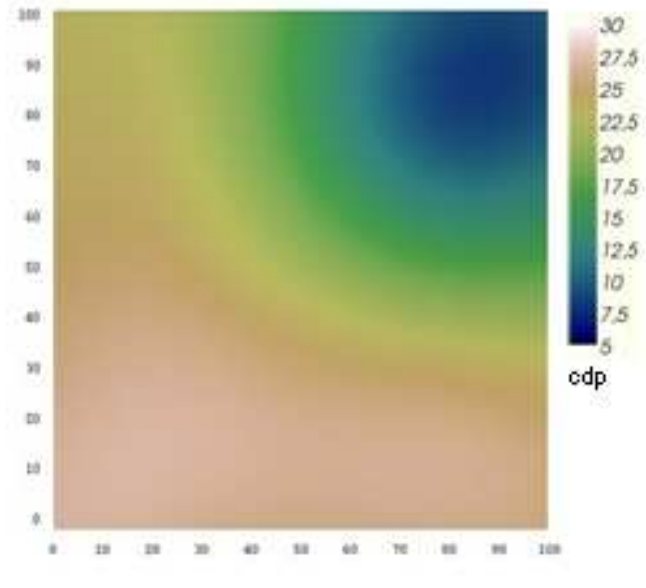

Fig_ch2_1b

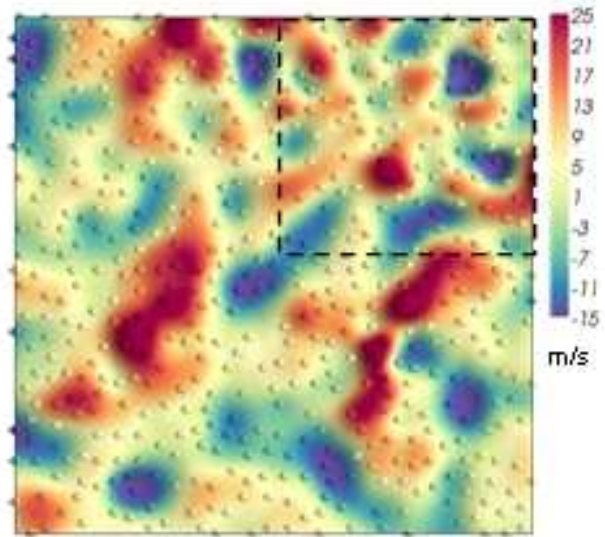

Fig_ch2_1d

Fig_ch2_1

Seismic data mapping (conventional and M-GS)

\subsection{Advantages}

M-GS ensures a better adequacy between the geostatistical model and the data. In consequence, spatial estimation and simulation results are more precise than those obtained with conventional variogram-based models. Regarding the previous seismic data mapping example, the improvement has been quantified through a cross-validation process. The M-GS map is on average $20 \%$ more precise than the conventional kriging map (Fig_ch2_1d) in the north-eastern part of the field. In other words the estimation errors have been reduced for $20 \%$. 
Moreover, M-GS opens the way to advanced geostatistical mapping (even simulating) practices by allowing the user to introduce his structural a priori knowledge about the data field directly into the spatial estimation model. In that way geostatistical mapping is no more a variogram guided process aiming at generating the most probable map, but a human process aiming at generating the most probable desired map. This last case is illustrated in Fig_ch2_2. A channel information, that should result from human interpretation, is introduced into the kriging model for mapping 25 depth data samples leading to a channel-driven map (Fig_ch2_2a) to be compared with a conventional global approach map (Fig_ch2_2b). The former presents a better continuity for the channel (red arrow) than the conventional which displays several individual depressions.

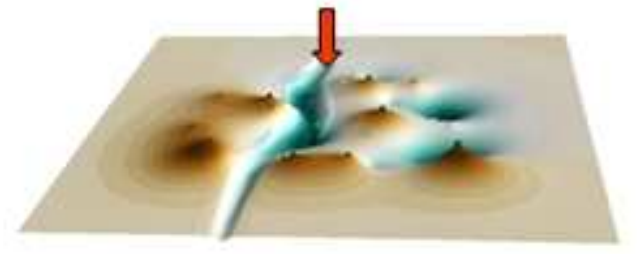

Fig_ch2_2a

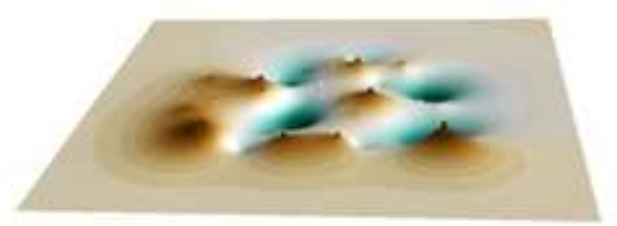

Fig_ch2_2b

Fig_ch2_2

M-GS guided mapping

\section{M-GS application to bathymetric mapping}

\subsection{Context}

The availability of accurate seafloor estimates is essential for numerous oceanographic projects, including hydrographic, oceanographic and biological models, sedimentary processes, seismic interpretation of buried channels or canyons, etc. Seafloor usually presents strong non stationarity and complex structures, such as small channels with varying orientations, spatially varying measurements errors, local heterogeneities for coastal areas, or deep canyons within general gentle slope for continental margins. 
Conventional variogram-based models often fail to produce consistent maps within such complex structural environment. More advanced models, such as MGS models, can be advantageously applied.

\subsection{Data set description}

Marenne-Oléron (West of France) is a semi-enclose Bay, first oyster farming zone in Europe. Shellfish culture activity induces silting on large intertidal mud and sandy-mud flats. Several channels incise the inlet between the coast line and Oléron Island. They are mainly controlled by strong tidal currents (up to 1.4 knots during the spring tides) with a residual ebb delta offshore the SW channel. The data set used in this work consists in more than two thousand sample points, organized along lines from West to East (Fig_ch3_1a). Samples are separated by few meters within lines. The (North-South) gap between two lines is about 100m. Data were acquired with a single beam echoes sounder for the monitoring of the evolution of the muddy layer.

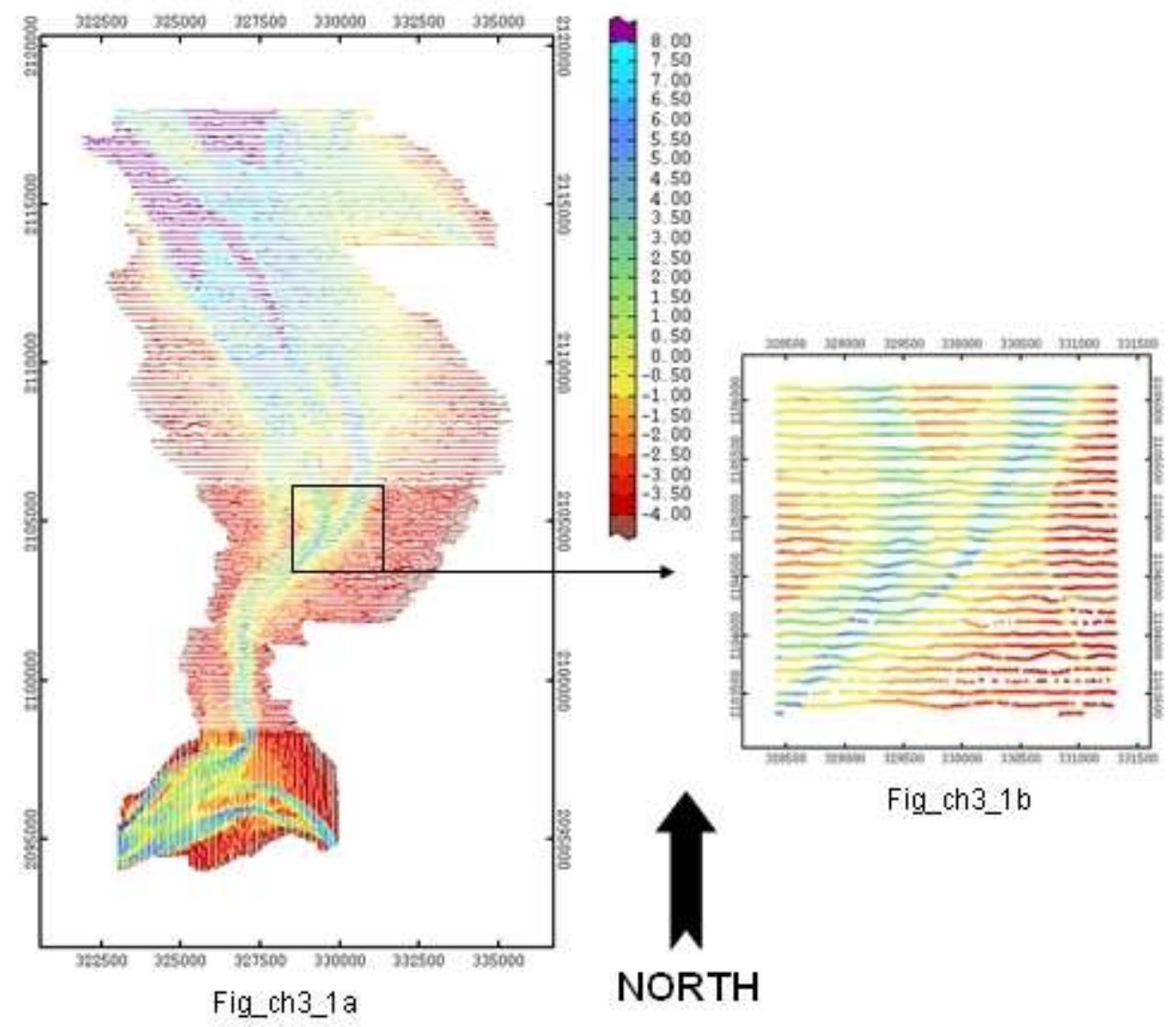

Fig_ch3_ 1

Marenne-Oléron data set 
A target area (Fig_ch3_1b and Fig_ch3_2) is selected for illustrating conventional and M-GS mapping results differences.

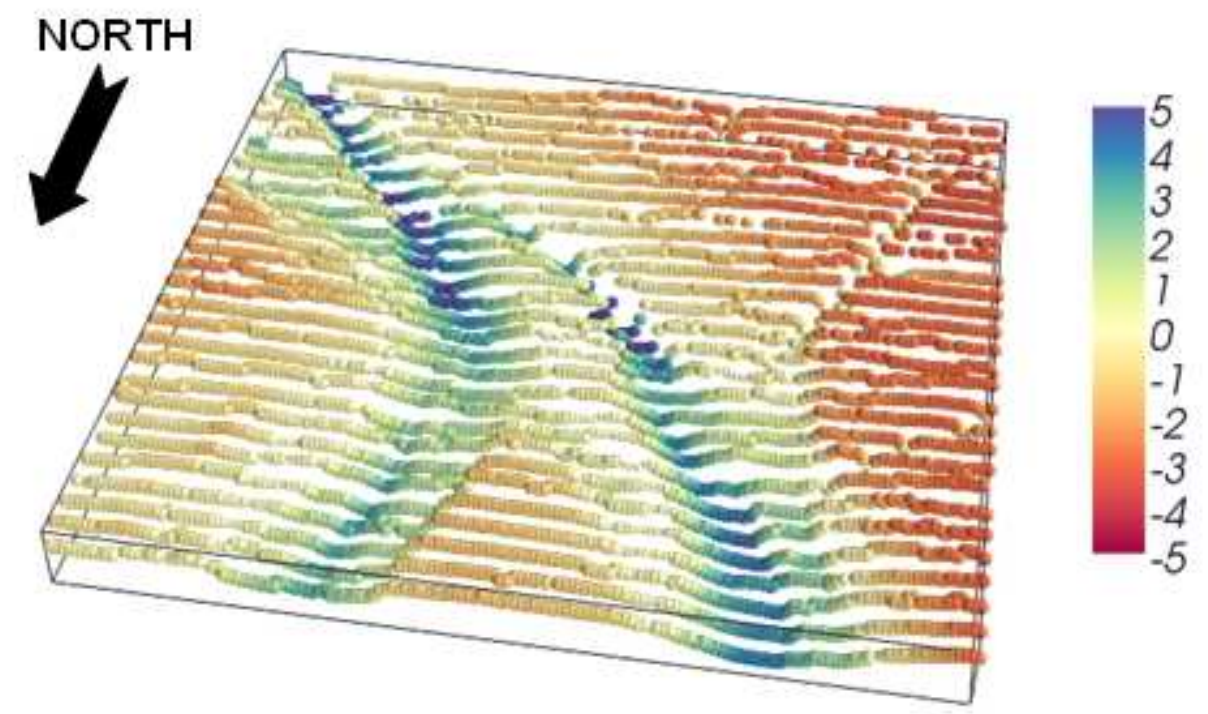

Fig_ch3_2

Target area

\subsection{Conventional variogram-based mapping}

For kriging purpose an experimental variogram is computed within the target area. An anisotropic spherical model (range 800m along X direction, 1200m along Y direction) is fitted to the experimental variogram (Fig_ch3_3) and used to map the depth data.

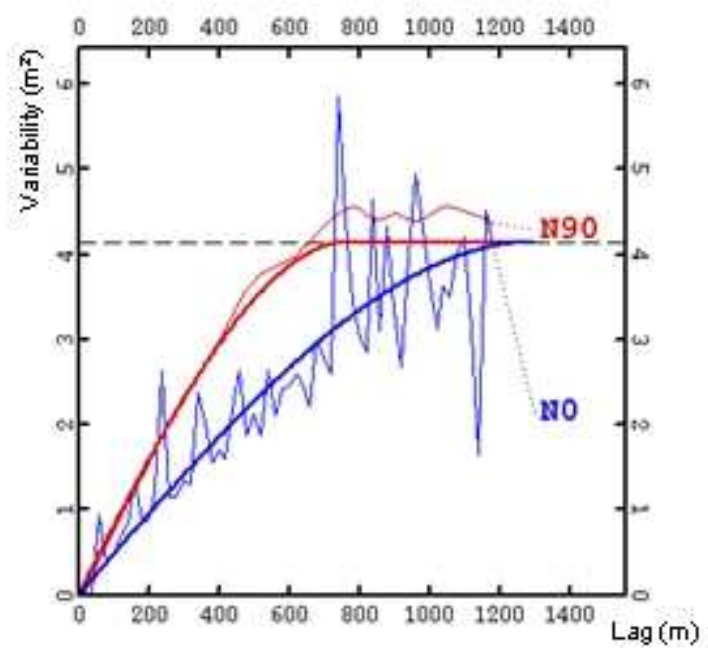

Fig_ch3_3

Global variogram modeling 
The resulting bathymetric map is shown on Fig_ch3_4. Major structures have been well imaged. However when looking into detail, the map contains some artefacts on the walls of the channels which are mainly due to the line-oriented organization of the data within strongly anisotropic areas. Moreover, one microchannel (red arrow), which is interpretable on the original data set, has not been reproduced at all.

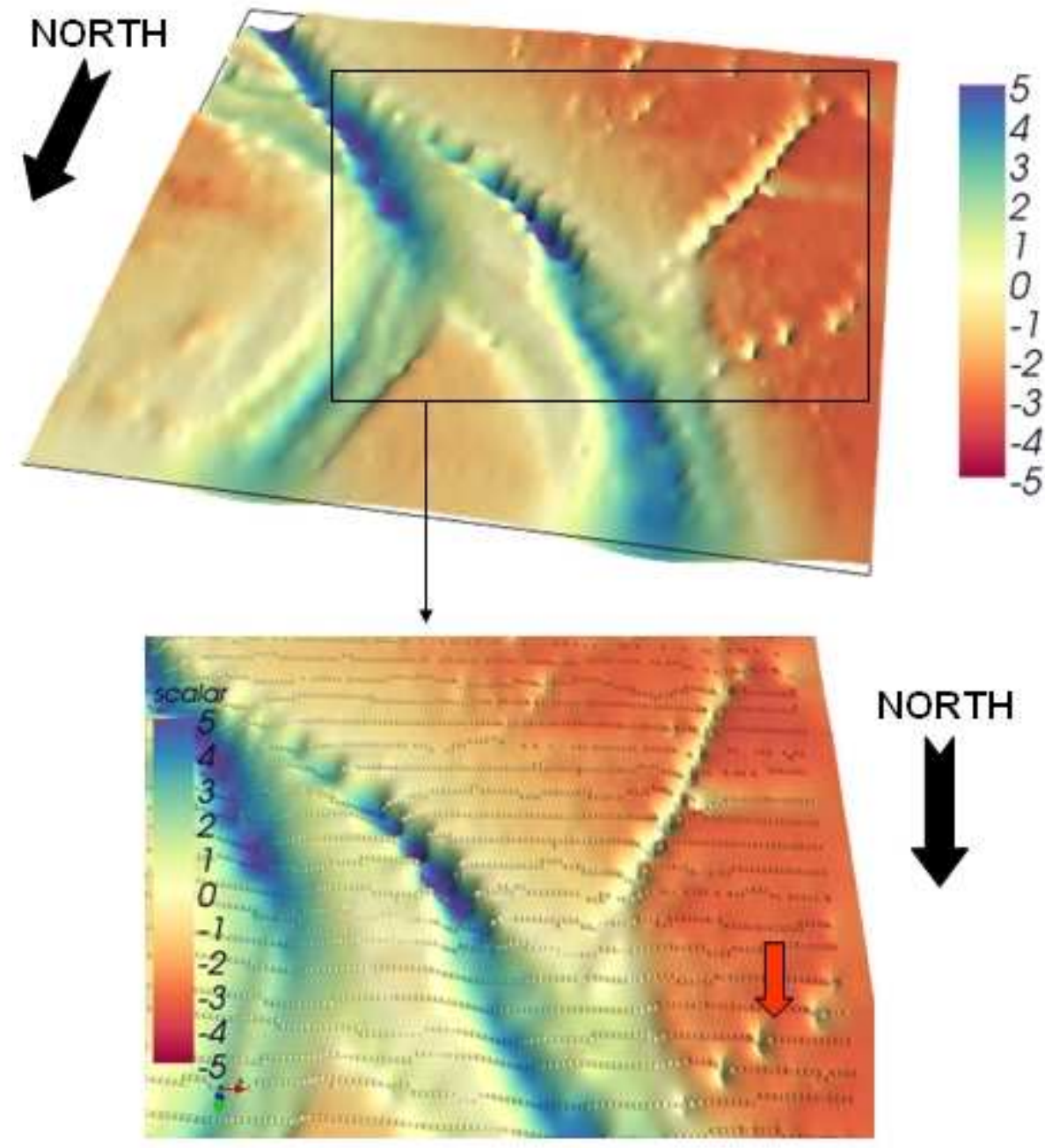

Fig_ch3_4

Global mapping results

Therefore, a more refined model is needed to attenuate the artefacts and to image correctly the interpreted micro-channel. 


\subsection{M-GS mapping}

The M-GS methodology enables to determine locally optimized structural and computational parameters. For the current application a specific emphasis is put on the range, the anisotropy and the related orientation of a generic spherical model. Firstly parameters are optimized during a M-structural analysis step, leading to several M-parameters maps. One resulting M-parameter map is shown in Fig_ch3_5a. This map illustrates the spatial variations of the shortest axe of the anisotropy ellipsoid. Afterwards prior knowledge is integrated into the model: additional information regarding the interpreted micro-channel is introduced into the M-parameters maps. The previous M-range map is transformed as shown in

\section{Fig_ch3_5b.}

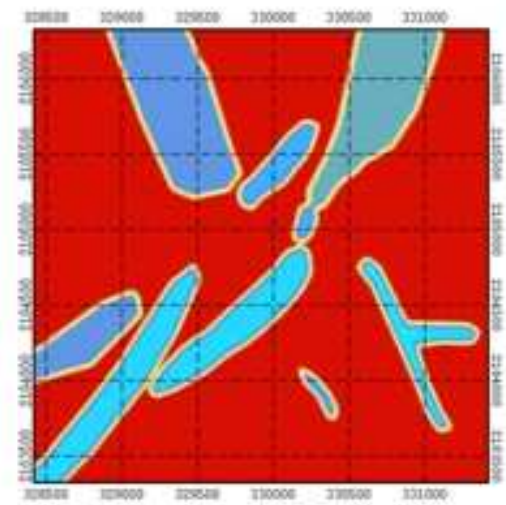

Fig_ch3_5a

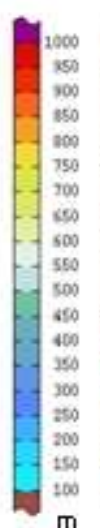

Fig_ch3_5

\section{Short range map}

Finally the M-parameters are used to estimate the bathymetry. Mapping results are displayed on Fig_ch3_6. The artefacts identified on the conventional map are no more visible and the interpreted micro-channel is imaged. In this case it is evident that the M-GS map is of better quality than the conventional map. 


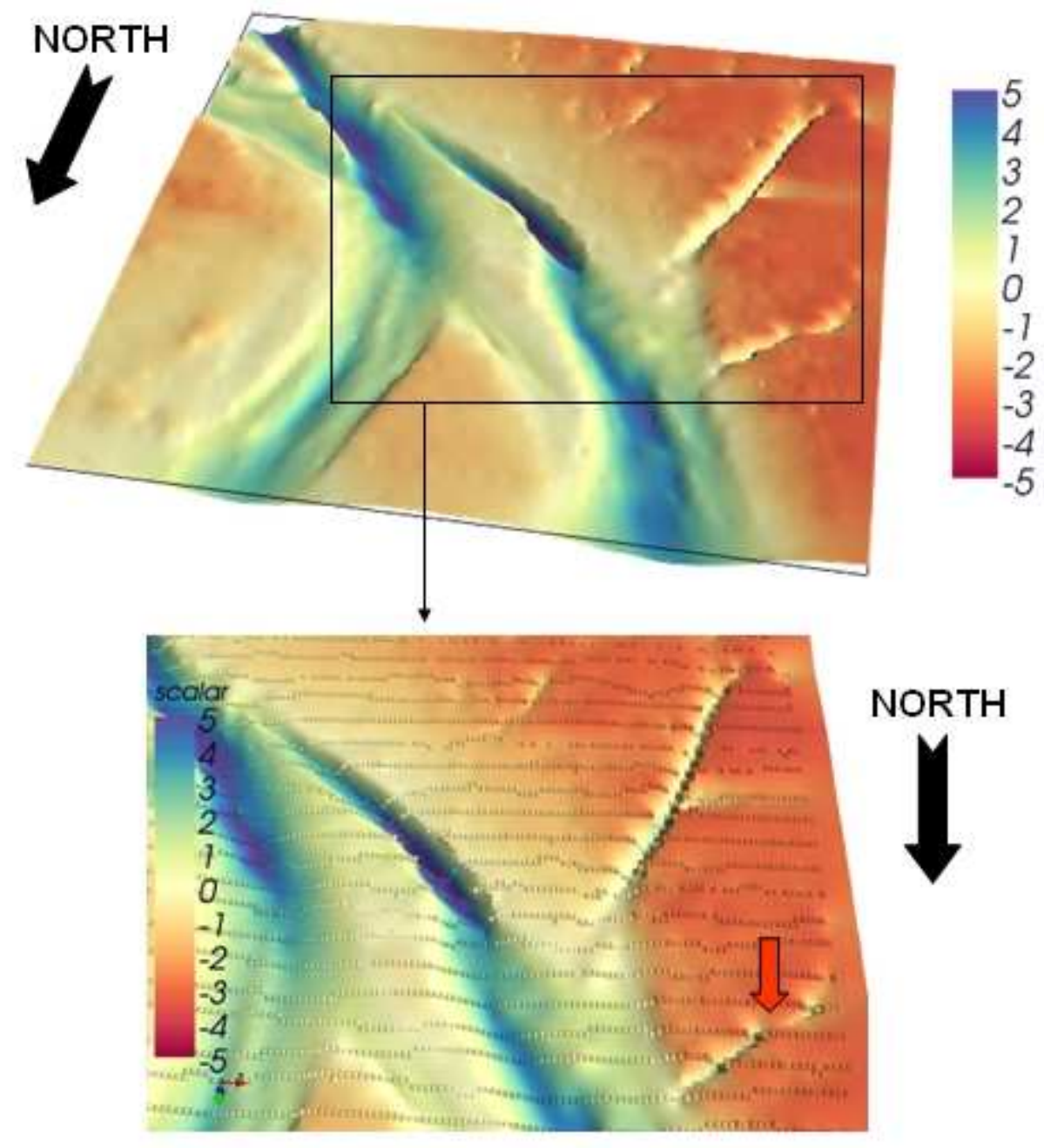

Fig_ch3_6

M-GS mapping results

\section{Conclusion}

The popularity of stationary variogram-based models is mainly explained by the easy interpretation which is made of the involved parameters. In particular, some structural parameters can be directly linked to the observation of the structural properties of the data. Advanced methodologies, which allow to manage spatial variations of these parameters, bring more accuracy to variogram-based models results, especially when processing large data sets and/or areas with complex structural patterns. 
In this direction, the M-GS methodology, which is dedicated to the optimization of variogram-based models parameters, is proved to be promising when applied to bathymetric or seismic interpretation data in a complex structural environment. The adequacy of the M-GS methodology in the framework of bathymetric mapping for Marenne-Oléron coast (West of France) is obvious. Moreover such methodology could be used to input different local structures into a general model in the aim of a regional synthesis.

\section{Acknowledgments}

The authors would like to thank Mr Fazilleau (Port Autonome de La Rochelle) and The "Conseil général de Charente Maritime" for having provided the Marenne-Oléron dataset.

\section{References}

Caetano H., Pereira M. J. and Guimaràes C. (2004), Use of Factorial Kriging to Incorporate Meteorological Information in Estimation of Air Pollutants. GeoENV IV Geostatistics for Environmental Applications, Part 2, Pages 55-65.

Chilès J.P. and Delfiner P. (1999), Geostatistics modeling spatial incertainty. Wiley series in probability and statistics.

Dubrule O. (2003), Geostatistics for seismic data integration in earth models. Distinguished Instructor Short Course - Distinguished Instructor series, $N^{\circ} 6$, SEG \& EAGE.

Matheron G. (1971), La théorie des functions aléatoires intrinsèques généralisées. Note Géostatistique $\mathrm{N}^{\circ} 117$. Technical report N-252. Centre de Géostatistique, Fontainebleau, France.

Pardo-Igúzquiza E. and Dowd P.A. (1998), The second-order stationary universal kriging model revisited. Mathematical Geology, Volume 30, Number 4, May 1998, pp. 347-378 (32). 